\title{
Infrared light enables visualization of the prostate carcinoma after radical prostatectomy
}

\author{
Besarion Partsvania ${ }^{1 *}$, Tamaz Sulaberidze ${ }^{1}$, Alexandre Khuskivadze², Levan Shoshiashvili ${ }^{3}$ and Ketevan Chubinidze ${ }^{3}$
}

*Correspondence: besari2@yahoo.com

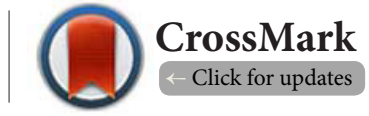

${ }^{1}$ Georgian Technical University, Institute of Cybernetics, Tbilisi, Georgia.

${ }^{2}$ Tbilisi Medical State University, Tbilisi, Georgia.

${ }^{3}$ Javakhishvili Tbilisi State University, Tbilisi, Georgia.

\begin{abstract}
Background: Imaging plays a crucial role in the identification, localization and grading of prostate carcinoma. However current imaging methods for the prostate cancer diagnosis are complicated and partially invasive. The drawback of existent imaging method is that they cannot detect prostate carcinoma at early stage of development. Therefore, a key challenge for prostate cancer detection is to use a simple and noninvasive method which will able to detect the cancer with very small dimensions. Correspondingly, the aim of present work was to demonstrate the possibility of using a near infrared light for the prostate cancer detection in vitro.
\end{abstract}

Methods: Experiments were carried out on prostates obtained after radical prostatectomy. Infrared light emitted diodes were used as illumination sources. Infrared light passing through the prostate was caught by charge-coupled device connected to computer and prostate infrared transillumination images were obtained.

Results: Intensity of near infrared light passing through the noncancerous prostate tissue is nearly homogeneous. Intensity of near infrared light passing through the cancerous outgrowth is lower than the intensity passing through the non-cancerous tissue of the same prostate, thereby cancerous formations are differentiated as the dark areas on the relatively white background. Specially developed software analyzes and processes distribution of intensities of the grayscale images, measures the ratio of their strength, and determines the rate of prostate malignancy.

Conclusions: Obtained results may hold some promise to make an important contribution to the diagnosis of prostate cancer in early stage of its development.

Keywords: Prostate cancer, near infra-red radiation, early diagnosis, transillumination imaging

\section{Introduction}

Prostate cancer after the lung cancer is the second cause of cancer death in men worldwide [1]. Just as with other cancers, timing and accuracy of diagnosis have major impacts on prostate cancer patient morbidity and quality of life. Although the imaging of prostate cancer has made tangible advances in recent years, the difficulty remains in identifying small volumes of prostate cancer in the prostate gland [2]. Efforts to utilize ultrasound for the detection of cancer have demonstrated poor specificity and poor predictive value. Within the current standard of care, transrectal ultrasound (TRUS) is used mainly to guide biopsies rather than to identify the location and extent of cancer [3]. The use of magnetic resonance imaging (MRI) alone to detect prostate cancer has been evaluated in a limited fashion. Newer imaging modalities such as endorectal coil MRI and magnetic resonance spectroscopic imaging (MRSI), offer the potential to detect prostate cancers [4]. However MRI uses a powerful magnetic field, radio frequency pulses and a computer to produce detailed pictures of the prostate. The strong magnetic field might be harmful. Besides, implanted medical devices that contain metal may malfunction or cause problems during an MRI exam [5,6]. Positron-emission tomography, is a technique that produces a three-dimensional image of functional processes in the body, and particularly in prostate. Three 
dimensional imaging is accomplished with the aid of computer tomography and X-ray scans $[7,8]$. However the $X$-ray scan contains some risk to be invasive. It is worth mentioning that these methods are extremely complicated. Besides, the methods are not able to detect very small prostate tumors. These limitations of the imaging methods of prostate cancer detection motivated to seek of alternative techniques. The use of near infrared NIR technology has garnered interest as a tool for intraoperative image-guided surgery in various surgical sub-disciplines for prostate [9-11]. In our previous works we show that NIR diaphanography technology could be applied for prostate cancer imaging in vitro $[12,13]$. However NIR technology is not used as imaging tool for prostate cancer detection in vivo yet. So, it is necessary to investigate the possibility of usage of IR radiation for cancer detection on isolated prostates.

In this paper, we demonstrate that NIR light can be used successfully for the visualization of cancerous outgrowth in the prostate, obtained by the radical prostatectomy.

\section{Materials and methods}

Experimental materials, such as prostate glands, were obtained from the radical prostatectomy. Light emitted diodes (LED)s (QT Brightek Company, USA) were utilized as the light sources, emitting an infrared light in the $850-900 \mathrm{~nm}$ range of the optical spectrum. The consuming power of LEDs was low, in the range of $0,08-0,14$ Watt and therefore, they did not cause any heating and damaging of the prostate tissues. To observe the prostate glands in the NIR spectrum IR-1000 CCD camera (Dage-MTI, USA) coupled with computer was used. Prostate is placed between CCD camera and LED Figure 1.

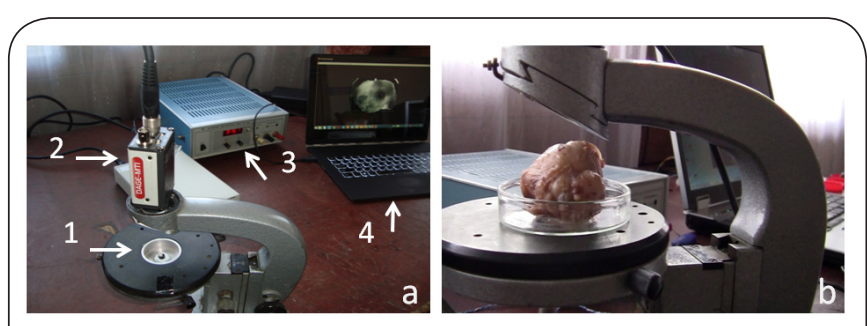

Figure 1. Experimental setup. In figure (a) by the arrows 1, 2, 3 and 4 are shown LED, CCD camera, power supply and computer, respectively. In the figure (b) is shown prostate placed on Petri dish between CCD camera and LED. NIR light irradiation angle of LED is not less than $130^{\circ}$. CCD camera capture area covers whole surface of a prostate exposed to it, thereby no scanning is necessary.

To avoid a possible artifact caused by the visible light, experiments were carried out in the darkness. In the first series of experiments LED was placed outside the prostate gland, from the bottom side, enabling NIR light to penetrate totally into prostate tissue. Whereas at another stage of the experiments, a LED was placed in the middle of the prostate, through the urethral channel, thereby CCD camera captured transillumination image of only the peripheral zone (rectum posteriorly part) of prostate tissue. This was circumstanced by the fact that prostate carcinoma mostly (more than 75\%) develops in this peripheral zone.

Biopsy on the patient was performed before surgery, which allowed us to preliminary define the location of cancerous outgrowth. In order to detect a location of cancerous outgrowth, the results obtained by the biopsy were compared to the experiments carried out by using of NIR light. Then, after the investigation in the infrared rays, the prostate glands were examined histo-morphologically [14] that enables a tumor to be located precisely in the prostate tissue. Because these investigations were carried out on the base of standard method, we considered not to include in our paper an illustrative stuff of the histo-morphological examinations. Accordingly, a location of each cancerous outgrowth was determined using two methods: one which was detected by histo-morphological examination and the other, by means of the NIR investigations.

To compare the transillumination images of non-cancerous tissues with the cancerous ones, we have elaborated software (computer program). The principle is: divides a brightness of the NIR transillumination image into 256 degrees. Consequently, the number 255 corresponds to the absolute white image, i.e., when NIR light enters CCD camera through a prostate without any absorption and on the contrary, 0 corresponds to the absolute dark image, i.e., when no NIR light enters CCD camera, i.e., when prostate absorbs entirely IR radiation. Then, on the NIR image potentially healthy and potentially malignant areas are marked. The program gives different codes to them. After that, program separately calculates the average values of the brightness for the potentially healthy tissue and for the malignant one, and calculates their ratio. Obtained data are stored in the memory. Such calculations are provided for all the next experiments so that, we can obtain the consequence of these ratios. When a number of experiments reach a statistically reliable value (at least 30 experiments), the program calculates a $95 \%$ confidence interval. In our case, after the examination of 31 cancerous prostates we obtained interval of the above mentioned ratios within 0.309839-0.333541 range. Then after, if new prostate NIR transillumination image will be analyzed, software will calculate above mentioned ratio of brightness. Finally, software will compare a meaning of this calculation with that confidence interval (0.309839-0.333541). If the newly measured number occurs in this $95 \%$ confidence interval, we can declare with $95 \%$ probability that this new prostate is cancerous. A similar procedures were taken place in the non-cancerous prostates, but, because in this case the infrared brightness of the images is almost the same everywhere, randomly selected and marked areas of the appropriate intensities are taken for the comparison of two meanings.

\section{Results and discussion}

In this investigation, we have carried out a series of experi- 
ments in which 31 cancerous and 30 non-cancerous prostate glands were examined. The infrared transillumination images of the non-cancerous prostates were characterized by a nearly homogeneous surface, in which a distribution of light intensities was equal everywhere. As shown in Figure 2, an entire brightness of prostate gland tissue with equal thickness is homogeneous and the distribution of light intensity is uniform in the corresponding areas. The rest 29 infrared images of the prostate gland are characterized similar optical properties, in which a prostate cancer has been detected neither before the prostatectomy, nor after the histo-morphological investigations. For the non-cancerous prostate, an above mentioned average ratio of intensities is equal to 0.938249 , which occurs in $95 \%$ confidence interval of $0.933605-0.942893$ range of ratios.

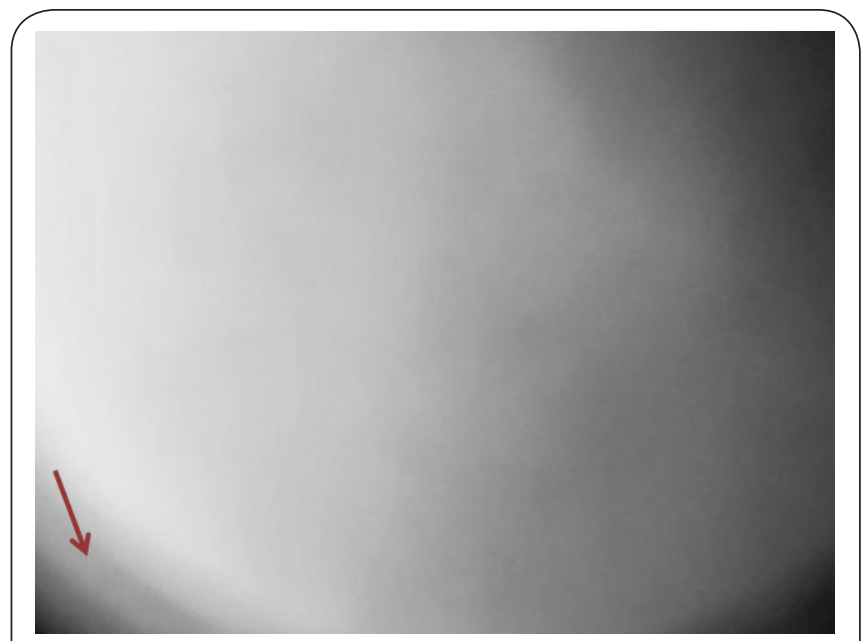

Figure 2. Infrared image of the non-cancerous prostate as it seen from the rectum side.

A radical prostatectomy was performed after the diagnosis of benign hyperplasia. Thereby the prostate was not cancerous. A prostate gland was placed on the Petri dish and an illumination was performed by using of LED with $850 \mathrm{~nm}$ wavelength. As the left side of a prostate was thinner than the right one, the left side in the picture looks brighter; however, both parts of the prostate gland can be considered as homogeneous. An inhomogeneity, shown by the arrow, belongs to the edge of Petri dish.

Further experiments, carried out on the cancerous prostate glands, have revealed that the cancerous formations are seen as the dark areas on the bright background. As an example, in Figure 3 it is shown an infrared image of cancerous prostate. This patient has prostate adenocarcinoma and was made a radical prostatectomy. Gleason score of the carcinoma was 8 . On the upper right side of the image is seen a dark area which is sharply distinguished from the surrounded areas having almost a homogeneous brightness. The aforementioned dark area coincided with the cancerous area predetermined by the biopsy, before the radical prostatectomy. A ratio of average intensities is equal to 0.321690 which occurs in $95 \%$

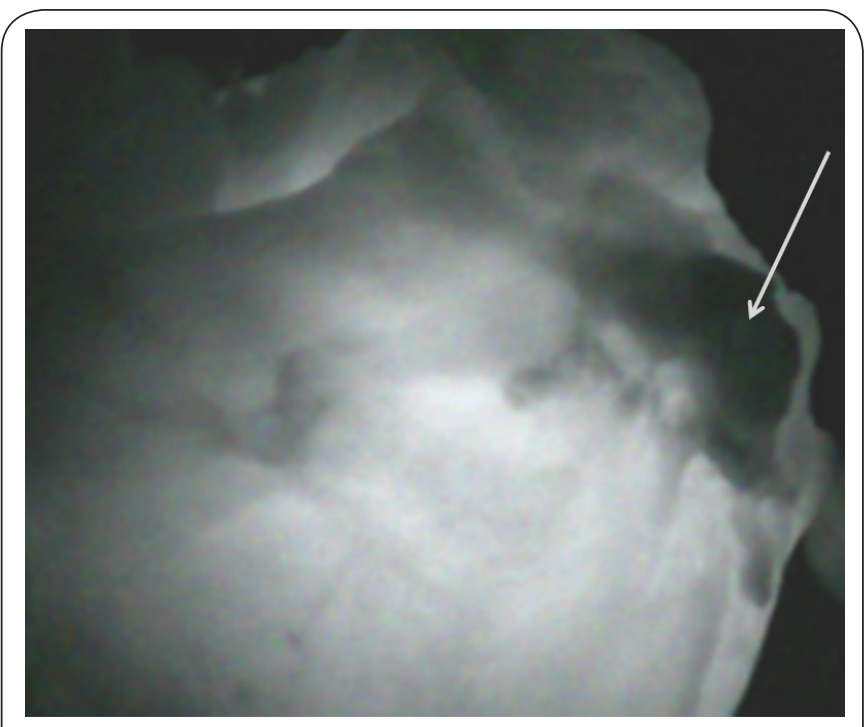

Figure 3. Infrared image of the malignant prostate gland. View from the rectum side. LED was placed inside the prostate through urethral channel. The dark area located in the upper right part of the picture (shown by arrow) corresponds to the cancerous outgrowth. This area is bounded by the less darken areas.

confidence interval of 0.309839-0.333541 range of ratios. After the NIR investigation, this prostate gland was examined hysto-morphologically, following the standard method. According to the hysto-morphological examination, there was a prostate adenocarcinoma. It is important to note that the location of cancerous areas for each suspected prostate gland, investigated by histo-morphological method and by the NIR examination coincided as well.

The transillumination images of prostates with different aggressiveness cancer are characterized by the different light transmission. Namely, the lower aggressiveness of the cancerous outgrowth, according to Gleason scope, the lower darkness of transillumination image was, as shown in Figure 4. A patient was diagnosed with the prostate carcinoma. Similar to the previous cases, the locations of the cancerous areas, determined by histo-morphological examination and by NIR investigation coincided. Numerical calculations have shown that, the ratio of the average value of intensities of the cancerous areas, to the average value of intensities of the non-cancerous areas, was equal to 0.310739 . This datum occurs within the $95 \%$ of the confidence interval for cancerous prostates. In fact, as it was shown by the histo-morphological investigations they were cancerous cells with Gleason score 5.

Besides, a developed program enables to map out the distribution of prostate carcinomas with different aggressiveness, in the color expression. In Figure $\mathbf{5}$ is demonstrated a part of the prostate gland in grayscale (a), and in color (b) representation.

So far a NIR transillumination technology widely was applied to the breast cancer diagnosis $[15,16]$. On the other hand, since the depth of NIR light penetration between a source placed 


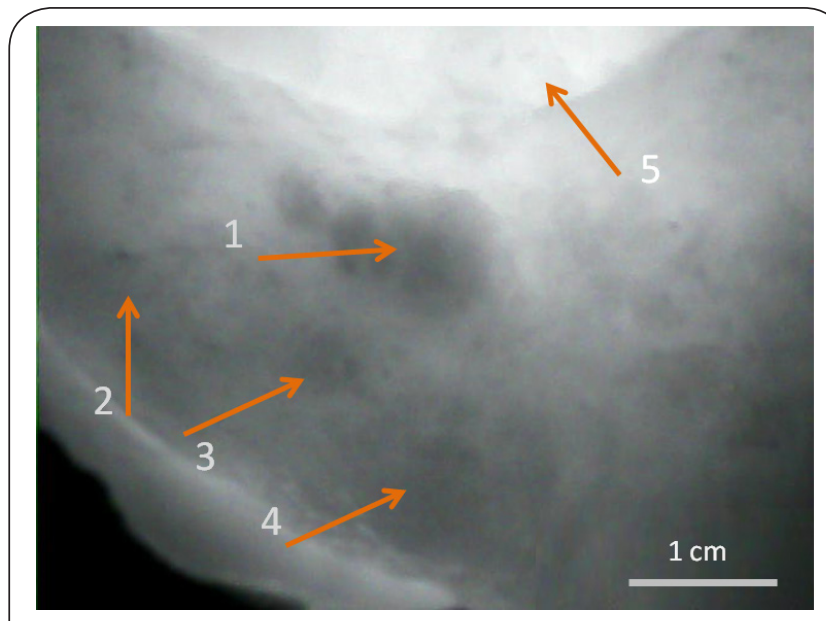

Figure 4. Infrared image of the prostate gland. A view from the rectum side. LED was placed into the prostate gland through the urethra channel. The transillumination images of the cancerous outgrows are represented as the acini of different sizes dark areas, located in different parts of the prostate gland (arrows 1, 2, 3, 4,). According to histo-morphological examinations, these areas were identified as the prostate adenocarcinoma as well. A relatively bright section, observed in the upper part of the picture (arrow 5) is the result of high intensity transmission, caused by the less thickness of prostate tissue in this area.

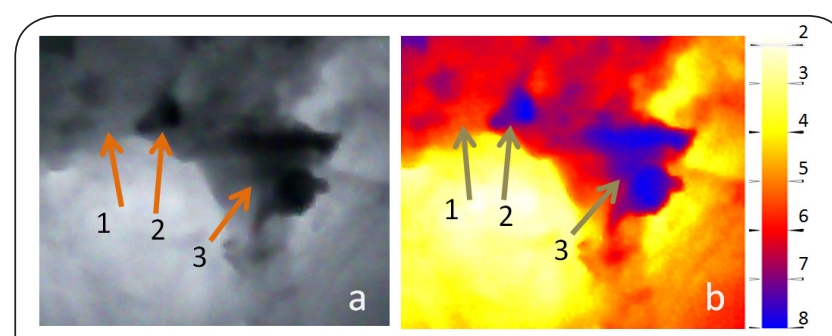

Figure 5. NIR image of the fragment of prostate gland in grayscale (a), and in color (b) representation.

As shown in the grayscale picture, the carcinomas with highest aggressiveness are the darkest areas indicated by the arrows 2 and 3. Less aggressiveness corresponds to the dark gray areas indicated by the arrow 1 . On the colored part of the picture, the carcinomas with highest aggressiveness are shown in blue (arrows 2 and 3), and the carcinomas with the less aggressiveness are shown in red (arrow 1). Right hand side of the picture represents the color bar, in which a blue color corresponds to the Gleason score with a value of 8 , red color corresponds to the Gleason score with a value of 6 , and yellow color corresponds to the non-cancerous portion respectively.

in the prostatic section of urethra and a camera placed in the rectum can be considerably shorter than that for a breast, actually a diaphanography imaging may work better for the prostate, than for the breast.

Our investigations have implied the demonstration whether the prostate cancer can exhibit particular optical properties upon exposure to infrared irradiation. The difference in the optical properties between the cancerous and non-cancerous cells can be caused due to the following reason: as it is well known, the cancerous cells are characterized by non-controlled and chaotic division. Normal cells have one nucleus and one nucleolus. Chromatins are thread like before the division. Cancerous cells have more than one large irregular shaped nucleus and nucleolus and condensed chromatins. Due to this reason a visible light penetration in normal and in cancerous cells is not the same, healthy cells are nearly transparent to light, whereas cancerous cells are much less transparent to light [17]. This decision is fair with respect to the NIR spectrum range, in which the biological tissues show very low absorption and autofluorescence. Aforementioned confirms that in NIR range the optical properties of cancerous tissue radically differ from that of non-cancerous tissue. In particular, the optical density of cancerous tissue is much higher, then the optical density of non-cancerous one. On the basis of this fact the samples were estimated visually, in particular, by using of NIR images were marked the malignant areas. Further histomorphologic investigations have up by nearly 100 percent confirmed the location of malignant areas estimated visually.

Since it was acquired some experience to estimate the level of samples disease in interactive regime, was developed a computer software, which in automatic regime determines the illness and degree of illness of the samples.

\section{Conclusion}

Thus, the evident advantage of the NIR method is its facility to detect an outgrowth as small as $5 \mathrm{~mm}$, for example, as shown in Figure 4, by the arrow 3. Another advantage of proposed method is that it can distinguish cancers with different aggressiveness. Besides, it has been shown that the proposed NIR method is a promising imaging modality in the field of prostate cancer targeting and visualization at the early stage of development. The minimized photo damage, low autofluorescence, high penetration depth and NIR LED based simple technique offers unique advantages for the visualization and diagnosis of prostate malignancy. However, it should be noted that the further studies are needed in order to identify and differentiate the infrared images of cancerous outgrowth with the other possible symptoms, such as prostatitis.

\section{Competing interests}

The authors declare that they have no competing interests.

\section{Authors' contributions}

\begin{tabular}{|l|c|c|c|c|c|}
\hline Authors' contributions & BP & TS & AK & LS & KC \\
\hline Research concept and design & $\checkmark$ & -- & -- & -- & -- \\
\hline Collection and/or assembly of data & $\checkmark$ & $\checkmark$ & $\checkmark$ & $\checkmark$ & $\checkmark$ \\
\hline Data analysis and interpretation & $\checkmark$ & $\checkmark$ & $\checkmark$ & $\checkmark$ & $\checkmark$ \\
\hline Writing the article & $\checkmark$ & $\checkmark$ & $\checkmark$ & $\checkmark$ & $\checkmark$ \\
\hline Critical revision of the article & $\checkmark$ & $\checkmark$ & $\checkmark$ & $\checkmark$ & $\checkmark$ \\
\hline Final approval of article & $\checkmark$ & $\checkmark$ & $\checkmark$ & $\checkmark$ & $\checkmark$ \\
\hline
\end{tabular}


Partsvania et al. Oncology Discovery 2016,

http://www.hoajonline.com/journals/pdf/2052-6199-4-2.pdf

doi: $10.7243 / 2052-6199-4-2$

\section{Acknowledgement}

This work was supported by ISTC foundation, project \#G-2188.

\section{Publication history}

Editor: Robert Amato, University of Texas Health Science Center, USA. EIC: Paul J. Higgins, Albany Medical College, USA.

Received: 07-Jul-2016 Final Revised: 19-Aug-2016

Accepted: 29-Aug-2016 Published: 09-Sep-2016

\section{References}

1. Siegel RL, Miller KD and Jemal A. Cancer statistics, 2015. CA Cancer J Clin. 2015; 65:5-29. | Article | PubMed

2. Taneja SS. Imaging in the diagnosis and management of prostate cancer. Rev Urol. 2004; 6:101-13. | PubMed Abstract | PubMed FullText

3. Harvey CJ, Pilcher J, Richenberg J, Patel U and Frauscher F. Applications of transrectal ultrasound in prostate cancer. Br J Radiol. 2012; 85 Spec No 1:S3-17. | Article | PubMed Abstract | PubMed FullText

4. Coakley FV, Qayyum A and Kurhanewicz J. Magnetic resonance imaging and spectroscopic imaging of prostate cancer. J Urol. 2003; 170:S69-75. | Article | PubMed

5. Formica $D$ and Silvestri S. Biological effects of exposure to magnetic resonance imaging: an overview. Biomed Eng Online. 2004; 3:11. | Article | PubMed Abstract | PubMed FullText

6. Bonekamp D, Jacobs MA, El-Khouli R, Stoianovici D and Macura KJ. Advancements in MR imaging of the prostate: from diagnosis to interventions. Radiographics. 2011; 31:677-703. | Article | PubMed Abstract | PubMed FullText

7. Oyama $\mathrm{N}$, Akino $\mathrm{H}$, Kanamaru $\mathrm{H}$, Suzuki $\mathrm{Y}$, Muramoto $\mathrm{S}$, Yonekura $\mathrm{Y}$, Sadato N, Yamamoto K and Okada K. ${ }^{11} \mathrm{C}$-acetate PET imaging of prostate cancer. J Nucl Med. 2002; 43:181-6. | Article | PubMed

8. Valk PE, Bailey DL, Townsend DW and Maisey MN. Positron Emission Tomography: Basic Science and Clinical Practice. New York: SpringerVerlag. 2004; 884.

9. Polom W, Markuszewski M, Rho YS and Matuszewski M. Usage of invisible near infrared light (NIR) fluorescence with indocyanine green (ICG) and methylene blue (MB) in urological oncology. Part 1. Cent European J Urol. 2014; 67:142-8. | Article | PubMed Abstract | PubMed FullText

10. Wang X, Huang SS, Heston WD, Guo H, Wang BC and Basilion JP. Development of targeted near-infrared imaging agents for prostate cancer. Mol Cancer Ther. 2014; 13:2595-606. | Article | PubMed Abstract I PubMed FullText

11. Zhu B, Wu G, Robinson H, Wilganowski N, Hall MA, Ghosh SC, Pinkston $\mathrm{KL}$, Azhdarinia A, Harvey BR and Sevick-Muraca EM. Tumor margin detection using quantitative NIRF molecular imaging targeting EpCAM validated by far red gene reporter iRFP. Mol Imaging Biol. 2013; 15:5608. | Article | PubMed

12. Khuskivadze A, Kochiashvili D and Partsvania B. Near Infrared Radiation in Diagnosis of Prostate Cancer: Preliminary Results. Urology. 2013; 82:S1-S334.

13. Khuskivadze A, Partsvania B and Kochiashvili D. Visualization of Human Prostate Cancer Using Infrared Radiation. Urology. 2014; 84:S304.

14. Svanholm H, Starklint H, Gundersen HJ, Fabricius J, Barlebo H and Olsen $\mathrm{S}$. Reproducibility of histomorphologic diagnoses with special reference to the kappa statistic. APMIS. 1989; 97:689-98. | Article | PubMed

15. Champaign $\mathrm{JL}$ and Cederbom GJ. Advances in breast cancer detection with screening mammography. Ochsner J. 2000; 2:33-5. | PubMed Abstract I PubMed FullText

16. Tromberg BJ, Cerussi A, Shah N, Compton M, Durkin A, Hsiang D, Butler $J$ and Mehta R. Imaging in breast cancer: diffuse optics in breast cancer: detecting tumors in pre-menopausal women and monitoring neoadjuvant chemotherapy. Breast Cancer Res. 2005; 7:279-85. | Article | PubMed Abstract | PubMed FullText

17. Ertefai $S$ and Profio AE. Spectral transmittance and contrast in breast diaphanography. Med Phys. 1985; 12:393-400. | Article I PubMed

\section{Citation:}

Partsvania B, Sulaberidze T, Khuskivadze A, Shoshiashvili $L$ and Chubinidze K. Infrared light enables visualization of the prostate carcinoma after radical prostatectomy. Oncol Discov. 2016; 4:2.

http://dx.doi.org/10.7243/2052-6199-4-2 